\title{
Epithelioid sarcoma is associated with a high percentage of SMARCB1 deletions
}

Lisa M Sullivan ${ }^{1}$, Andrew L Folpe ${ }^{2}$, Bruce R Pawel ${ }^{1}$, Alexander R Judkins ${ }^{3}$ and Jaclyn A Biegel ${ }^{1,4}$

${ }^{1}$ Department of Pathology and Laboratory Medicine, The Children's Hospital of Philadelphia, Perelman School of Medicine of the University of Pennsylvania, Philadelphia, PA, USA; ${ }^{2}$ Department of Laboratory Medicine and Pathology, Mayo Clinic, Rochester, MN, USA; ${ }^{3}$ Department of Pathology and Laboratory Medicine, Children's Hospital Los Angeles, Keck School of Medicine University of Southern California, Los Angeles, CA, USA and ${ }^{4}$ Department of Pediatrics, The Children's Hospital of Philadelphia, Perelman School of Medicine of the University of Pennsylvania, Philadelphia, PA, USA

SMARCB1 gene alterations were first described in highly malignant rhabdoid tumors of the kidney, brain (atypical teratoid/rhabdoid tumor) and soft tissue. An increasing number of tumors have now shown loss of SMARCB1 protein expression by immunohistochemistry, including the majority of epithelioid sarcomas. However, investigations of SMARCB1 gene alterations in epithelioid sarcoma have produced conflicting results. The aim of this study was to evaluate SMARCB1 status using Sanger sequencing of the coding region and multiplex ligation-dependent probe amplification, a rapid and sensitive method for detecting intragenic deletions and duplications, which has not been used in previous studies. Twenty-one epithelioid sarcomas of both classical and proximal type were selected for SMARCB1 gene testing and SMARCB1 immunohistochemistry. Nineteen of $21(90 \%)$ epithelioid sarcomas were SMARCB1 negative by immunohistochemistry. Twelve of the $19(63 \%)$ had adequate DNA recovery for evaluation. Ten of $12(83 \%)$ tumors showed homozygous deletions of the gene. Two cases showed heterozygous deletions and polymorphisms, but no sequence mutations. These results confirm the high frequency of SMARCB1 deletions in epithelioid sarcoma and show that multiplex ligation-dependent probe amplification is a reliable method for detection of deletions in these cases, which can be performed on formalin-fixed, paraffin-embedded tissue. Given the high percentage of SMARCB1 alterations in epithelioid sarcoma, these findings argue against using SMARCB1 gene deletion as a tool in distinguishing them from malignant rhabdoid tumors.

Modern Pathology (2013) 26, 385-392; doi:10.1038/modpathol.2012.175; published online 12 October 2012

Keywords: epithelioid sarcoma; malignant rhabdoid tumor; SMARCB1

Malignant rhabdoid tumor is a highly aggressive childhood neoplasm that occurs in the kidney, liver, soft tissue and central nervous system. Classically, these tumors exhibit varying numbers of rhabdoid cells with eccentric nuclei containing prominent nucleoli and abundant cytoplasm with eosinophilic globular cytoplasmic inclusions. The diagnosis of malignant rhabdoid tumor was revolutionized by the discovery of SMARCB1 alterations in these tumors with subsequent loss of protein expression

Correspondence: Dr LM Sullivan, MD, Department of Anatomic Pathology, The Children's Hospital of Philadelphia, CHOP Main 5NW26, 34th Street and Civic Center Blvd., Philadelphia, PA 19104, USA.

E-mail: sullivanl@email.chop.edu

Received 16 May 2012; revised 4 September 2012; accepted 4 September 2012; published online 12 October 2012 detectable by immunohistochemistry. ${ }^{1-4}$ Comprehensive genomic analysis using high-density singlenucleotide polymorphism-based oligonucleotide arrays, multiplex ligation-dependent probe amplification and coding sequence analysis has shown that $98 \%$ of malignant rhabdoid tumor, atypical teratoid/rhabdoid tumor and extrarenal rhabdoid tumors have bi-allelic SMARCB1 alterations with few additional karyotypic abnormalities. ${ }^{5,6}$

It is becoming increasingly clear that loss of SMARCB1 protein expression is not exclusively limited to malignant rhabdoid tumor, as an increasing number of tumors have been described, which are also negative for SMARCB1 by immunohistochemistry. Soft-tissue tumors comprise the majority of cases, and include epithelioid sarcoma, schwannomas associated with germline SMARCB1 abnormalities, and a subset of cases diagnosed as 
epithelioid malignant peripheral nerve sheath tumors, pediatric undifferentiated sarcomas, synovial sarcomas, myoepithelial carcinomas and extraskeletal myxoid chondrosarcomas. ${ }^{6-12}$ Although the percentage of negative cases varies by tumor type, both proximal and classical type epithelioid sarcomas have consistently shown loss of staining in the majority of cases. ${ }^{8}$

Classical and proximal type epithelioid sarcomas occur most often in adolescents and young adults as compared with early childhood for malignant rhabdoid tumor. Classical type epithelioid sarcoma is composed of plump epithelioid to spindled cells and usually exhibits a nodular growth pattern with hyalinized collagen and central necrosis, at times simulating a benign necrobiotic process. Proximal type epithelioid sarcoma is most often seen in the pelvis, perineal region and genital tract. Histologically, it is characterized by large cells with epithelioid cytomorphology, marked cytologic atypia with frequent rhabdoid features and lack of a granulomalike pattern. ${ }^{13}$

Regardless of the classical or proximal type designation, these tumors express epithelial and mesenchymal markers with loss of SMARCB1, reminiscent of malignant rhabdoid tumor. ${ }^{8,13,14}$ However, the expression of CD34 in approximately half of all epithelioid sarcomas may aid in their distinction from malignant rhabdoid tumor, which are CD34 negative. ${ }^{14,15}$ Although many malignant rhabdoid tumors and epithelioid sarcomas can be distinguished based on anatomic location, histologic features, patient demographics and CD34 immunoreactivity, this is not true in all instances. Particularly challenging are tumors in older children and adolescents in axial locations, which show overlapping features of malignant rhabdoid tumor and proximal epithelioid sarcoma. Although some may argue that proximal epithelioid sarcoma and malignant rhabdoid tumor represent different manifestations of the same tumor, differential CD34 expression and the rarity of epithelioid sarcoma in adolescents and young adults with germline SMARCB1 mutations support these tumors as distinct entities. ${ }^{6,14,15}$ Furthermore, malignant rhabdoid tumors most often show isolated $22 \mathrm{q}$ abnormalities, whereas many proximal and classical epithelioid sarcomas have chromosome 22 abnormalities in addition to a more complex karyotype including abnormalities on chromosomes $6,7,8,14$, 18,20 and $21.5,16-18$

Investigations of SMARCB1 gene alterations in epithelioid sarcoma have shown conflicting results. Previously reported data from three studies that cumulatively included 65 SMARCB1 negative tumors by immunohistochemistry showed that the percentage of cases with homozygous SMARCB1 deletions ranged from 5 to $71 \% \cdot{ }^{19-21}$ Of note, these investigators employed a range of techniques including combinations of fluorescence in situ hybridization, array comparative genomic hybridization and quantitative PCR. In addition to chromosome 22 deletions, there are also three reports of SMARCB1inactivating mutations in epithelioid sarcoma, including two frameshift and one nonsense mutations. ${ }^{20,22}$

None of the previous studies used multiplex ligation-dependent probe amplification, which allows for multiple specific nucleic acid sequences to be amplified simultaneously using a single PCR. Multiplex ligation-dependent probe amplification for the nine exons of SMARCB1 as well as probes proximal and distal on $22 \mathrm{q}$ is currently used as a clinical test for the diagnosis of malignant rhabdoid tumor at our institution. The advantage of this method is that it is rapid, with an overnight turn around time. It is also the most sensitive method for detecting $0,1,2$ or 3 copies of a given target sequence in a DNA specimen. ${ }^{5}$ Commercial probe mixes (MRC-Holland, Amsterdam, the Netherlands) allow for the interrogation of 45-50 sequences simultaneously. In most cases, formalin-fixed, paraffin-embedded tissue can be used in this assay.

The aim of this study was to better establish the percent and spectrum of SMARCB1 gene abnormalities in epithelioid sarcoma, using sequencing to identify mutations, and multiplex ligation-dependent probe amplification for the detection of small deletions and duplication within the SMARCB1 gene. ${ }^{5,23}$ The SMARCB1 results for epithelioid sarcoma were compared with previously published data on malignant rhabdoid tumor.

\section{Materials and methods}

\section{Cases}

This study was conducted under the auspices of The Children's Hospital of Philadelphia and Mayo Clinic Institutional Review Boards approval for the use of human tissues. Twenty-one formalin-fixed, paraffinembedded epithelioid sarcoma cases diagnosed between 1994 and 2008 were selected from the files of the Department of Pathology and Laboratory Medicine, Mayo Clinic and used for SMARCB1 gene testing and immunohistochemistry. Routine hematoxylin and eosin staining, SMARCB1 immunohistochemistry and pathology reports were reviewed for each tumor to confirm the diagnosis and ensure adequate tissue for examination. Patient demographic and SMARCB1 staining information are shown in Table 1.

\section{Immunohistochemical Staining and Scoring}

SMARCB1 antibody (BAF47, BDBioscience 612110) staining, deparaffinization, epitope retrieval with pH 9 buffer and immunohistochemistry at an antibody concentration of 1:100 were performed on the Leica Bond-Max Autostainer using the Bond Polymer Refine Detection System (Leica Microsystems, 
Table 1 Epithelioid sarcoma: patient demographics and SMARCB1 staining

\begin{tabular}{|c|c|c|c|c|c|}
\hline Case & Diagnosis & Sex & $\begin{array}{c}\text { Age at diagnosis } \\
\text { (years) }\end{array}$ & Location & SMARCB1 \\
\hline 1 & Classical & Female & 40 & Hand & Negative \\
\hline 2 & Classical & Male & 18 & Leg & Negative \\
\hline 3 & Proximal & Male & 17 & Perineum & Negative \\
\hline 4 & Classical & Female & 40 & Hand & Negative \\
\hline 5 & Proximal & Male & 45 & Unknown & Negative \\
\hline 6 & Proximal & Male & 37 & Unknown & Negative \\
\hline 7 & Classical & Male & 42 & Arm & Negative \\
\hline 8 & Classical & Female & 40 & Hand & Negative \\
\hline 9 & Classical & Male & 58 & Arm & Negative \\
\hline 10 & Proximal & Male & 49 & Groin & Negative \\
\hline 11 & Proximal & Male & 37 & Inguinal & Negative \\
\hline 12 & Classical & Male & 32 & Shoulder & Negative \\
\hline 13 & Proximal & Male & 20 & Scrotum & Negative \\
\hline 14 & Classical & Female & 24 & Thigh & Negative \\
\hline 15 & Classical & Female & 25 & Hand & Negative \\
\hline 16 & Classical & Male & 38 & Forearm & Negative \\
\hline 17 & Classical & Male & 18 & Shin & Negative \\
\hline 18 & Classical & Male & 56 & Arm & Negative \\
\hline 19 & Classical & Male & 22 & Forearm & Negative \\
\hline 20 & Proximal & Male & 48 & Buttock & Positive \\
\hline 21 & Classical & Female & 72 & Foot & Positive \\
\hline
\end{tabular}

Bannockburn, IL). Nuclear staining for SMARCB1 was evaluated as positive (normal) or negative. Nonneoplastic tissue and infiltrating lymphocytes on the slide served as internal positive controls.

\section{SMARCB1 Gene Evaluation}

Cases were selected for DNA analysis if they were SMARCB1 negative by immunohistochemistry, and if there were available scrolls of formalin-fixed, paraffin-embedded tissue composed of at least $50 \%$ tumor based on review of corresponding hematoxylin and eosin-stained slides. DNA was extracted from scrolls using the RecoverAll Total Nucleic Acid Isolation Kit for formalin-fixed, paraffin-embedded tissues (Applied Biosystems/Ambion, Austin, TX) according to the manufacturer's protocol. DNA was then quantitated using a Nanodrop ND-1000 UV-Vis Spectrophotometer (Nanodrop Technologies, Wilmington, DE). Multiplex ligation-dependent probe amplification was performed with genomic DNA using the SALSA MLPA P258 (SMARCB1) kit (MRCHolland) according to previously published methods based on the manufacturer's protocol. ${ }^{5}$ Briefly, the kit contains 2 probes for each of the 9 exons of $S M A R C B 1$, probes for 8 other genes on chromosome 22 , and 14 control probes from other chromosomes. The 8 other genes on chromosome 22 include DGCR8, SNAP29, LZTR1, PPIL2, GNAZ, SNRPD3, SEZ6L and NIPSNAP1. The samples were processed and data analyzed as previously described. ${ }^{24}$

PCR-based mutation analysis was performed as reported previously using primers for exons 1-9 of the SMARCB1 gene. ${ }^{1}$ PCR products for individual exons were sequenced utilizing the BigDye
Terminator v3.1 Cycle Sequencing Kit from Applied Biosystems as per the manufacturer's protocol. Sequencing products were analyzed on a 3730 DNA Analyzer (Applied Biosystems/Ambion) by The Children's Hospital of Philadelphia Nucleic Acid/Protein Core.

\section{Results}

\section{Immunohistochemical Staining in Epithelioid Sarcomas}

Results of the SMARCB1 immunohistochemistry staining are shown in Table 1 . Nineteen of 21 cases $(90 \%)$ were negative for SMARCB1, which is similar to previously published data. ${ }^{8}$ Figure 1 shows examples of SMARCB1-negative and -positive cases.

\section{SMARCB1 Gene Alterations}

Twelve of the 19 (63\%) SMARCB1-negative cases had an adequate tumor percentage and adequate DNA recovery to perform multiplex ligation-dependent probe amplification and sequence analysis. Results are shown in Table 2. Ten of the cases (83\%) were characterized by homozygous deletions of at least two exons within the SMARCB1 gene. Figure 2 demonstrates multiplex ligation-dependent probe amplification results from case 15 with a homozygous deletion. The remaining two cases (16\%) showed heterozygous deletions of all nine exons. PCR-based sequencing of the two cases with loss of only one copy of SMARCB1 failed to show a mutation in the coding region of the gene, and thus the second inactivating event was not identified.

\section{Discussion}

Although approximately $98 \%$ of malignant rhabdoid tumors harbor bi-allelic SMARCB1 gene abnormalities with few additional karyotypic abnormalities, there is conflicting data for epithelioid sarcoma. ${ }^{5,19-22}$ The first major study to evaluate SMARCB1 in epithelioid sarcoma was by Modena et al in 2005. ${ }^{19}$ Of 7 (5 proximal and 2 classical) immunohistochemistry-negative cases, $5(71 \%)$ proximal epithelioid sarcomas demonstrated homozygous deletions of SMARCB1 using bacterial artificial chromosome probes for dual-color fluorescence in situ hybridization. Two of the cases with homozygous deletions were also tested using comparative genomic hybridization, with one case showing a heterozygous deletion and the other failing to demonstrate any deletions. The discrepancy in the fluorescence in situ hybridization and comparative genomic hybridization results was likely due to the comparative genomic hybridization resolution of $2-4 \mathrm{Mb}$ in this study. Semi-quantitative PCR of SMARCB1 exon 1 was performed on four of the 

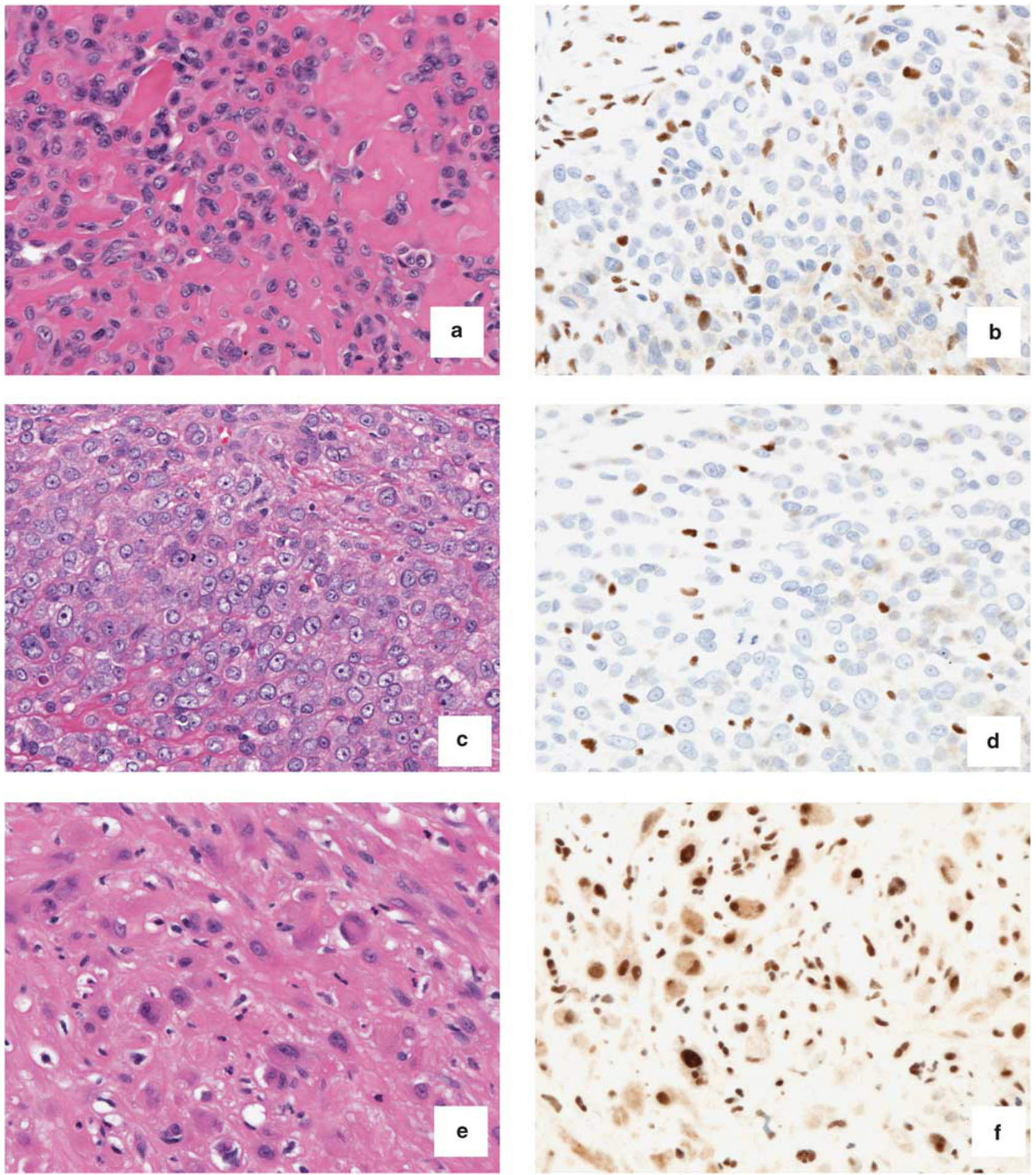

Figure 1 Examples of epithelioid sarcomas: classical epithelioid sarcoma showing (a) intersecting hyalinized collagen (hematoxylin and eosin, $\times 40)$ and (b) negative SMARCB1 staining in tumor cells with positive infiltrating lymphocytes $(\times 40)$. Proximal epithelioid sarcoma showing (c) confluent growth of large epithelioid cells with prominent nucleoli (hematoxylin and eosin, $\times 40)$ and $(\mathbf{d})$ negative SMARCB1 staining in tumor cells with positive background endothelial cells $(\times 40)$. Proximal epithelioid sarcoma showing $(\mathbf{e})$ pleomorphic cells with a mixed spindle and epithelioid morphology (hematoxylin and eosin, $\times 40$ ) and (f) retained SMARCB1 expression $(\times 40)$.

cases with homozygous deletions and showed concordant results. No mutations were identified by sequencing.
In 2009 Kohashi et $a l^{20}$ studied 39 immunohistochemistry-negative epithelioid sarcomas (19 proximal and 27 classical). Deletions were identified 
Table 2 SMARCB1 gene alterations in 12 epithelioid sarcomas

\begin{tabular}{|c|c|c|c|}
\hline Case number & Subtype & Multiplex ligation-dependent probe amplification & Sequencing \\
\hline 2 & Classical & Homozygous deletion, exons 1-9 & Not done \\
\hline 3 & Proximal & Homozygous deletion, exons 1-9 & Not done \\
\hline 5 & Proximal & Homozygous deletion, xons 1-9 & Not done \\
\hline 6 & Proximal & Heterozygous deletion, exons 1-9 & $\begin{array}{l}\text { Intron } 5 \text { polymorphisms: } \\
\text { c. } 628+66 \mathrm{G}>\mathrm{C} \\
\text { c. } 628+118 \mathrm{C}>\mathrm{T}\end{array}$ \\
\hline 7 & Classical & Homozygous deletion, exons 1-9 & Not done \\
\hline 9 & Classical & Homozygous deletion, exons 1-9 & Not done \\
\hline 10 & Proximal & Homozygous deletion, exons $4-5$ & Not done \\
\hline 11 & Proximal & Homozygous deletion, exons 6-9 & Not done \\
\hline 12 & Classical & Heterozygous deletion, exons 1-9 & $\begin{array}{l}\text { Intron } 5 \text { polymorphisms: } \\
\text { c. } 628+66 \mathrm{G}>\mathrm{C} \\
\text { c. } 628+118 \mathrm{C}>\mathrm{T} \\
\text { Exon } 7 \text { polymorphism: } \\
\text { c. } 897 \mathrm{G}>\mathrm{A} \\
\text { Intron } 7 \text { polymorphism: } \\
\text { c. } 986+56 \text { +986 + 57dupAA }\end{array}$ \\
\hline 15 & Classical & Homozygous deletion, exons 1-9 & Not done \\
\hline 17 & Classical & Homozygous deletion, exons 1-9 & Not done \\
\hline 18 & Classical & Homozygous deletion, exons 1-9 & Not done \\
\hline
\end{tabular}
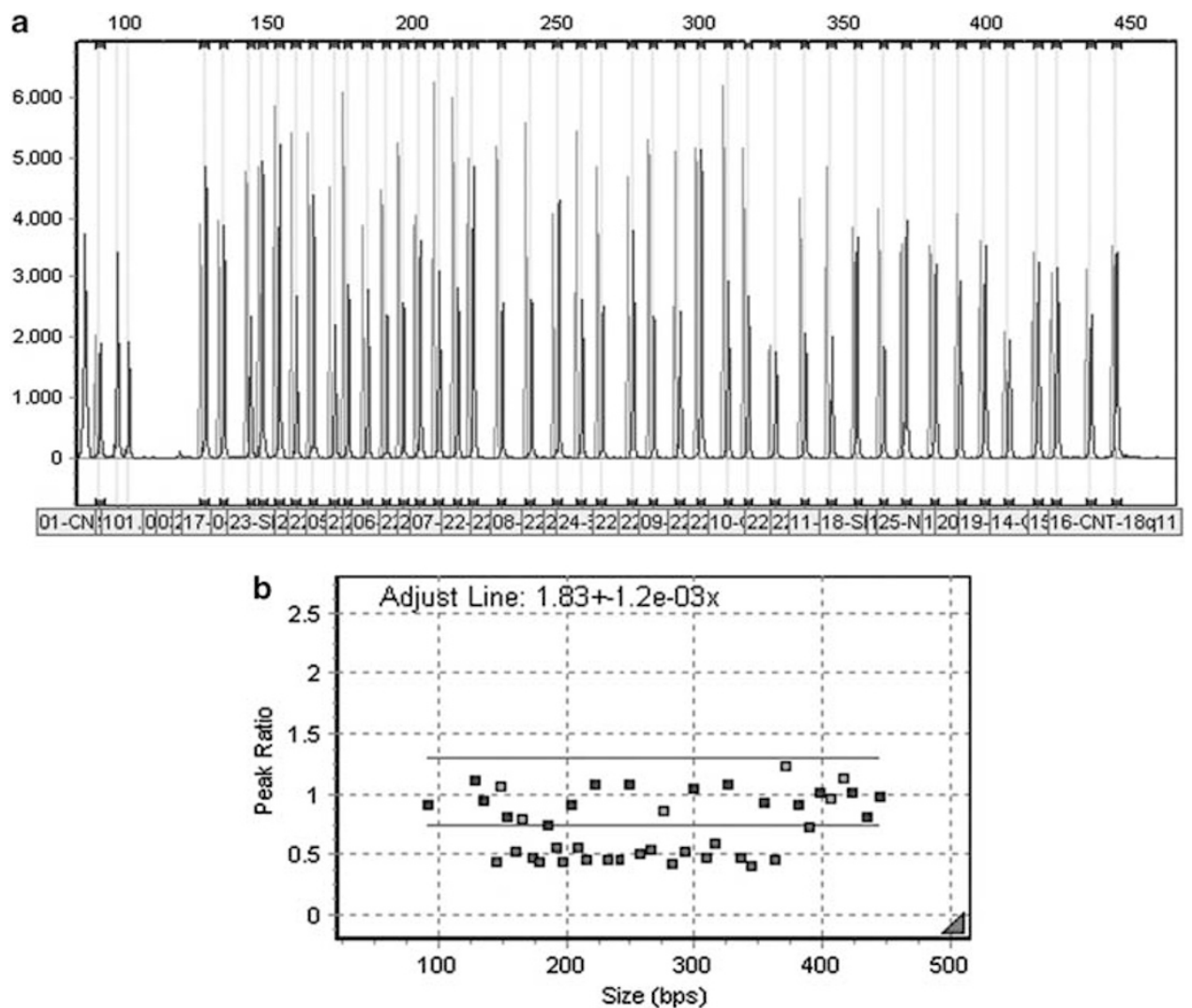

Figure 2 Multiplex ligation-dependent probe amplification for the SMARCB1 locus, case 15. Capillary gel electrophoresis (a) shows red peaks corresponding to the size standards for the PCR products and blue peaks corresponding to all PCR-amplified products including the exons of the SMARCB1 gene, loci on chromosome 22q proximal and distal to SMARCB1 and internal control probes on additional chromosomes throughout the genome. Comparing the capillary gel electrophoresis peak pattern obtained on normal reference samples with that obtained in the tumor, the relative quantity of each amplicon can then be determined. This peak ratio graph (b) is approximately 1 for each internal control probe (blue) as well the majority of reference probes on 22q proximal and distal to SMARCB1 (green). The peak ratios for the GNAZ locus (proximal to SMARCB1), and the probes for exons 1-9 of SMARCB1 (red), fall below 0.5, demonstrating that less than half of the expected amount of DNA is present for the SMARCB1 gene exons. Taking into account the normal stromal contamination in the sample, this is consistent with a homozygous deletion. 
by quantitative real-time PCR for exons 1-9 of the SMARCB1 gene. Only two (5\%) proximal epithelioid sarcoma cases showed homozygous deletions. Sequencing was performed for mutation analysis and two (5\%) proximal epithelioid sarcomas showed homozygous frameshift mutations consisting of a one base pair deletion in exon 9 and a two base pair deletion in exon 3 . Following this article, a correspondence was written by Flucke et $a l^{22}$ describing a case of proximal epithelioid sarcoma with a c.769C $>$ T mutation in exon 6 resulting in the generation of an in-frame stopcodon.

Most recently in 2011, Gasparini et al ${ }^{21}$ evaluated 19 immunohistochemistry-negative epithelioid sarcomas (5 proximal and 14 classical). As tabulated in the article, a total of 11 cases (58\%, 4 proximal and 7 classical) were shown to harbor homozygous deletions of SMARCB1 with 9 identified through fluorescence in situ hybridization and an additional 2 by quantitative PCR for exon 4 . No mutations were identified through sequencing. This was the first series to demonstrate SMARCB1 deletions in both proximal and classical epithelioid sarcoma.

Taken together, the above studies demonstrate that SMARCB1 deletions are far more common than gene mutations in epithelioid sarcoma; however, the exact number of cases with primary gene abnormalities varies dramatically between published reports. In the present study, 12 immunohistochemistrynegative epithelioid sarcomas (7 classical, 5 proximal) had at least $50 \%$ tumor in the sample and adequate DNA recovery for multiplex ligationdependent probe amplification and sequencing. Multiplex ligation-dependent probe amplification revealed that $10 / 12$ (83\%, 6 classical, 4 proximal) harbored homozygous deletions. This percentage is similar to what we reported for extrarenal rhabdoid tumors but higher than that seen in CNS atypical teratoid/rhabdoid tumor or renal malignant rhabdoid tumor. ${ }^{6}$ Of note, 2 of the 10 cases $(20 \%)$ had a homozygous deletion spanning a small portion of the gene, either exons $4-5$ or exons $6-9$. These cases would likely have been missed using traditional fluorescence in situ hybridization techniques or limited quantitative PCR including only a subset of exons. Of the two remaining cases $(17 \%$, one classical, one proximal), multiplex ligation-dependent probe amplification showed heterozygous deletions involving all exons of the SMARCB1 gene. No mutations were identified by sequencing.

The finding of this high rate of bi-allelic loss or inactivation of SMARCB1 in a high percentage of both proximal and classical epithelioid sarcoma tumors tested is consistent with the two hit model of carcinogenesis associated with loss of function of a tumor suppressor gene, and correlates with the near universal bi-allelic loss or inactivation of SMARCB1 seen in malignant rhabdoid tumor. Although two of our cases only showed heterozygous deletions, the loss of SMARCB1 protein expression suggests the presence of an undetected abnormality of SMARCB1 in the other allele. Possible explanations for the failure to demonstrate this may be due to the high percentage of normal stromal cells in the samples, because a high percentage of normal cell contamination can mask a deletion using multiplex ligation-dependent probe amplification. Despite the fact that hematoxylin and eosin-stained sections of each tumor were evaluated for percentage of tumor present, increased proportions of normal cells could have been present in the tissue scrolls used for DNA extraction. As a result, the two cases with heterozygous deletions may represent false-negative results in tumors with true homozygous deletions. One of the two cases had additional formalin-fixed, paraffin-embedded tissue available for fluorescence in situ hybridization testing, which would have allowed for evaluation of SMARCB1 copy number limited exclusively to the tumor tissue. However, the fluorescence in situ hybridization assay was unsuccessful. Alternatively, there may have been an intronic mutation present that would have been missed by only analyzing the exons and intron/exon boundaries.

It is important to note that the high percentage of cases with homozygous deletions, relatively low number of cases with intragenic deletions and subset of cases where only a single gene defect could be identified, raises the possibility that loss of SMARCB1 protein expression may be a secondary event in epithelioid sarcoma. The driving force for chromosome 22 deletions seen in epithelioid sarcoma may actually result from selection of a nearby gene instead of SMARCB1. In addition, an intronic or upstream change that affects expression of the SMARCB1 protein also cannot be excluded based on our data.

The results from this study and others indicate that somatic SMARCB1 status alone is not sufficient to differentiate malignant rhabdoid tumor from epithelioid sarcoma. As a result, the finding of biallelic mutations or deletions in SMARCB1 should not be used as a criteria to support reclassifying epithelioid sarcoma as malignant rhabdoid tumor. Being able to reliably differentiate epithelioid sarcoma and malignant rhabdoid tumor is important due to differences in treatment, prognosis and the association of malignant rhabdoid tumor with germline mutations necessitating additional genetic testing and family counseling. ${ }^{6,25-27}$ Despite intensive treatment for patients with malignant rhabdoid tumor, many patients die of widespread metastatic disease, especially those with germline mutations and second primary lesions. For example, one recent study limited to extrarenal non-cerebral rhabdoid tumors demonstrated a median time to recurrence of 5 months with only 1 of 26 (4\%) patients alive and without evidence of disease at 7 years. ${ }^{28}$ Currently, many patients with soft tissue malignant rhabdoid tumor are enrolled in Children's Oncology Group protocol AREN0321, which employee 
a highly aggressive initial multimodality approach including surgery, radiation therapy and multiagent chemotherapy using vincristine, doxorubicin, cyclophospamide, carboplatin and etoposide, but results of patient outcome are unknown at this time.

In contrast, the overall 5-year survival rates for classical epithelioid sarcoma range from 50 to $85 \%$ and 10-year survival rates range from 42 to $55 \% .{ }^{18}$ The propensity of epithelioid sarcoma to grow along fascial planes, tendons and nerves often results in initial resections with positive margins and a protracted clinical course including multiple local recurrences and metastasis. ${ }^{26}$ Adequate treatment for epithelioid sarcoma requires early radical local excision or amputation with regional lymph node dissection. The specific regimen for radiation therapy and adjuvant chemotherapy is unclear, but is often similar to treatment plans for other adult sarcomas. ${ }^{29,30}$

The survival rate for proximal epithelioid sarcoma is challenging to define due to difficulties in separating these lesions from malignant rhabdoid tumor in many studies and the fact that many of the reported adverse prognostic features for classical epithelioid sarcoma are often seen in proximal epithelioid sarcoma, including non-distal extremity location, large tumor size, increased tumor depth and inadequate initial excision. ${ }^{26,27,31}$ Until recently, many used SMARCB1 deletions or absence of SMARCB1 protein expression by immunohistochemistry as evidence for the diagnosis of malignant rhabdoid tumor, especially in children. However, studies of firmly diagnosed epithelioid sarcoma, including those in older patients, have shown SMARCB1 abnormalities as well, suggesting that SMARCB1 status alone cannot reliably distinguish epithelioid sarcoma from malignant rhabdoid tumor. ${ }^{19-21}$

Our study demonstrates that the majority of both proximal and classical epithelioid sarcoma have homozygous deletions of SMARCB1, which can be identified with multiplex ligation-dependent probe amplification. Given the high percentage of SMARCB1 alterations in epithelioid sarcoma, these findings argue against using SMARCB1 gene deletion to distinguish epithelioid sarcoma from malignant rhabdoid tumor. ${ }^{20}$ Moreover, these findings suggest an underlying genetic relationship between epithelioid sarcoma and malignant rhabdoid tumor. Further study is warranted to explore why these two tumors which so closely share phenotypic and genetic features behave in clinically distinctive ways.

\section{Acknowledgements}

This work was supported in part by a grant from the National Institutes of Health (CA46274) to Jacyln A Biegel, PhD. We thank Laura Tooke for assistance with the multiplex ligation-dependent probe amplification image.

\section{Disclosure/conflict of interest}

The authors declare no conflict of interest.

\section{References}

1 Biegel JA, Zhou JY, Rorke LB, et al. Germ-line and acquired mutations of INI1 in atypical teratoid and rhabdoid tumors. Cancer Res 1999;59:74-79.

2 Versteege I, Sevenet N, Lange J, et al. Truncating mutations of hSNF5/INI1 in aggressive paediatric cancer. Nature 1998;394:203-206.

3 Hoot AC, Russo P, Judkins AR, et al. Immunohistochemical analysis of hSNF5/INI1 distinguishes renal and extra-renal malignant rhabdoid tumors from other pediatric soft tissue tumors. Am J Surg Pathol 2004; 28:1485-1491.

4 Judkins AR, Mauger J, Ht A, et al. Immunohistochemical analysis of hSNF5/INI1 in pediatric CNS neoplasms. Am J Surg Pathol 2004;28:644-650.

5 Jackson EM, Sievert AJ, Gai X, et al. Genomic analysis using high-density single nucleotide polymorphismbased oligonucleotide arrays and multiplex ligationdependent probe amplification provides a comprehensive analysis of INI1/SMARCB1 in malignant rhabdoid tumors. Clin Cancer Res 2009;15:1923-1930.

6 Eaton KW, Tooke LS, Wainwright LM, et al. Spectrum of SMARCB1/INI1 mutations in familial and sporadic rhabdoid tumors. Pediatr Blood Cancer 2011;56:7-15.

7 Cheng JX, Tretiakova M, Gong C, et al. Renal medullary carcinoma: rhabdoid features and the absence of INI1 expression as markers of aggressive behavior. Mod Pathol 2008;21:647-652.

8 Hornick JL, Dal Cin P, Fletcher CD. Loss of INI1 expression is characteristic of both conventional and proximal-type epithelioid sarcoma. Am J Surg Pathol 2009;33:542-550.

9 Kreiger PA, Judkins AR, Russo PA, et al. Loss of INI1 expression defines a unique subset of pediatric undifferentiated soft tissue sarcomas. Mod Pathol 2009;22:142-150.

10 Gleason BC, Fletcher CD. Myoepithelial carcinoma of soft tissue in children: an aggressive neoplasm analyzed in a series of 29 cases. Am J Surg Pathol 2007; 31:1813-1824.

11 Kohashi K, Oda Y, Yamamoto H, et al. Reduced expression of SMARCB1/INI1 protein in synovial sarcoma. Mod Pathol 2010;23:981-990.

12 Trobaugh-Lotrario AD, Tomlinson GE, Finegold MJ, et al. Small cell undifferentiated variant of hepatoblastoma: adverse clinical and molecular features similar to rhabdoid tumors. Pediatr Blood Cancer 2009; 52:328-334.

13 Guillou L, Wadden C, Coindre JM, et al. 'Proximaltype' epithelioid sarcoma, a distinctive aggressive neoplasm showing rhabdoid features. clinicopathologic, immunohistochemical, and ultrastructural study of a series. Am J Surg Pathol 1997;21:130-146.

14 Chbani L, Guillou L, Terrier P, et al. Epithelioid sarcoma: a clinicopathologic and immunohistochemical analysis of 106 cases from the french sarcoma group. Am J Clin Pathol 2009;131:222-227.

15 Oda Y, Tsuneyoshi M. Extrarenal rhabdoid tumors of soft tissue: Clinicopathological and molecular genetic review and distinction from other soft-tissue sarcomas with rhabdoid features. Pathol Int 2006;56:287-295. 
16 Lualdi E, Modena P, Debiec-Rychter M, et al. Molecular cytogenetic characterization of proximal-type epithelioid sarcoma. Genes Chromosomes Cancer 2004;41:283-290.

17 Feely MG, Fidler ME, Nelson M, et al. Cytogenetic findings in a case of epithelioid sarcoma and a review of the literature. Cancer Genet Cytogenet 2000;119: 155-157.

18 Weiss S, Goldblum R(eds) Enzinger \& Weiss's soft tissue tumors; 5th edn., Mosby Elsevier: China; 2008, pp 1191-1203.

19 Modena P, Lualdi E, Facchinetti F, et al. SMARCB1/ INI1 tumor suppressor gene is frequently inactivated in epithelioid sarcomas. Cancer Res 2005;65:4012-4019.

20 Kohashi $\mathrm{K}$, Izumi $\mathrm{T}$, Oda $\mathrm{Y}$, et al. Infrequent SMARCB1/INI1 gene alteration in epithelioid sarcoma: a useful tool in distinguishing epithelioid sarcoma from malignant rhabdoid tumor. Hum Pathol 2009; 40:349-355.

21 Gasparini P, Facchinetti F, Boeri M, et al. Prognostic determinants in epithelioid sarcoma. Eur J Cancer 2011;47:287-295.

22 Flucke U, Slootweg PJ, Mentzel T, et al. Re: Infrequent SMARCB1/INI1 gene alteration in epithelioid sarcoma: a useful tool in distinguishing epithelioid sarcoma from malignant rhabdoid tumor: direct evidence of mutational inactivation of SMARCB1/INI1 in epithelioid sarcoma. Hum Pathol 2009;40:1361 2; author reply $1362-4$.
23 den Dunnen JT, White SJ. MLPA and MAPH: sensitive detection of deletions and duplications. Curr Protoc Hum Genet 2006; Chapter 7:Unit 7.14.

24 Jalali GR, Vorstman JA, Errami A, et al. Detailed analysis of 22q11.2 with a high density MLPA probe set. Hum Mutat 2008;29:433-440.

25 de Visscher SA, van Ginkel RJ, Wobbes $\mathrm{T}$, et al. Epithelioid sarcoma: still an only surgically curable disease. Cancer 2006;107:606-612.

26 Chase DR, Enzinger FM. Epithelioid sarcoma. diagnosis, prognostic indicators, and treatment. Am J Surg Pathol 1985;9:241-263.

27 Casanova M, Ferrari A, Collini P, et al. Italian Soft Tissue Sarcoma Committee. Epithelioid sarcoma in children and adolescents: a report from the italian soft tissue sarcoma committee. Cancer 2006;106: 708-717.

28 Bourdeaut F, Lequin D, Brugieres L, et al. Frequent hSNF5/INI1 germline mutations in patients with rhabdoid tumor. Clin Cancer Res 2011;17:31-38.

29 Murray PM. Soft tissue sarcoma of the upper extremity. Hand Clin 2004;20:325-333; vii.

30 Whitworth PW, Pollock RE, Mansfield PF, et al. Extremity epithelioid sarcoma. Amputation vs local resection. Arch Surg 1991;126:1485-1489.

31 Callister MD, Ballo MT, Pisters PW, et al. Epithelioid sarcoma: results of conservative surgery and radiotherapy. Int J Radiat Oncol Biol Phys 2001;51: 384-391. 